\title{
LEP searches in R-parity Violation scenarios
}

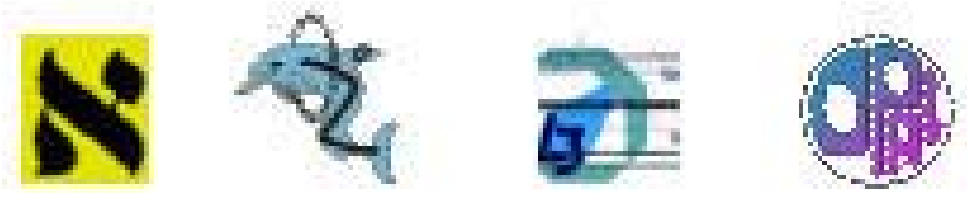

Theodora D. Papadopoulou

NTU Athens

July 25 - Amsterdam ICHEP 2002 
- Introduction

- RPV Superpotential

- Decays via trilinear RPV Couplings

- Search strategies

- Review of Updated Analyses Results

- Exclusion plots and limits

- Summary

- Conclusion

- As an Epilogue 
- What is Rp and why go beyond ?

$>$ a discrete multiplicative symmetry in SUSY models connected to matter parity

$$
R_{p}=(-1)^{2 S+3 B+L}
$$

$\mathrm{R}_{p}=1$ for $\mathbf{S M}$ particles

$\mathrm{R}_{p}=-1 \quad$ for SUSY particles

o SUSY particles produced in pairs

o LSP is stable

o experimental signature of SUSY $\mathbf{E}_{\mathrm{T}}$ miss

$\longrightarrow$ fast proton decay is suppressed 
- Rp can be explicitly broken by trilinear terms in the superpotential

$$
\begin{gathered}
\mathrm{W}=\lambda_{\mathrm{ijk}} \mathrm{L}_{\mathrm{i}} \mathrm{L}_{\mathrm{j}} \overline{\mathrm{E}}_{\mathrm{k}} \\
\Delta \mathrm{L} \neq 0 \\
9 \text { Couplings }(i \neq j)
\end{gathered}
$$

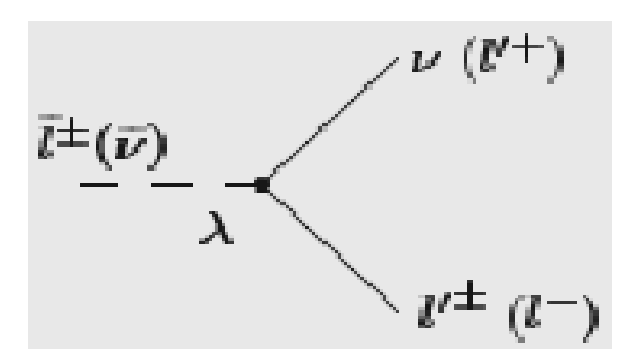$$
+\lambda_{i j k}^{\prime} L_{i} Q_{j} \bar{D}_{k}
$$$$
+\lambda_{i j k}^{\prime \prime} \overline{\mathrm{U}}_{\mathrm{i}} \overline{\mathrm{D}}_{\mathrm{j}} \overline{\mathrm{D}}_{\mathrm{k}}
$$

$$
\Delta \mathrm{L} \neq 0
$$

27 Couplings

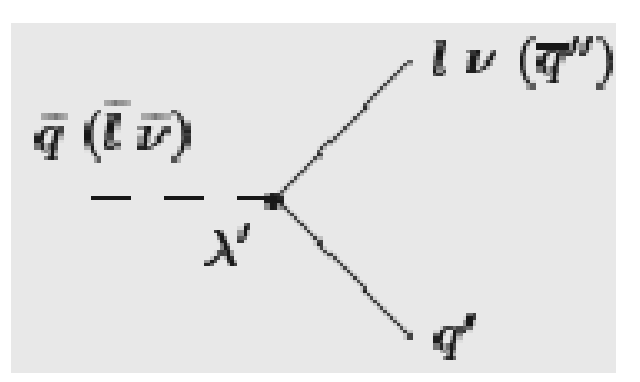

$\Delta \mathrm{B} \neq 0$

9 Couplings $(j \neq k)$

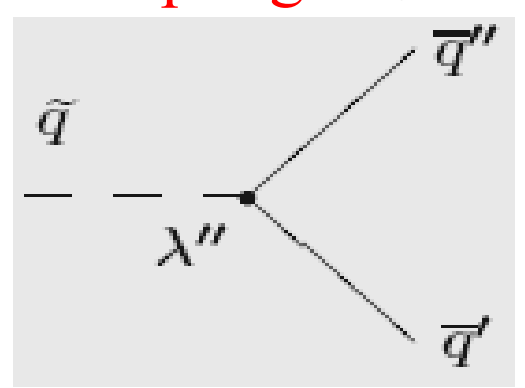

o single sparticle production via a $\Delta \mathrm{L} \neq 0$ or a $\Delta \mathrm{B} \neq 0$ operator

o Unstable LSP !

o Signature of multilepton or multijet events in excess

$\longrightarrow$ fast proton decay is suppressed if Lepton and Baryon number Violating Couplings are not simultaneously present 
Decay topologies

$9 \lambda_{i j k}+27 \lambda_{i j k}^{\prime}+9 \lambda^{\prime \prime}{ }_{i j k}=45$ new couplings

Hierarchies in RpV Couplings expected ( as for Yukawa Couplings generating fermion masses)

- direct decays

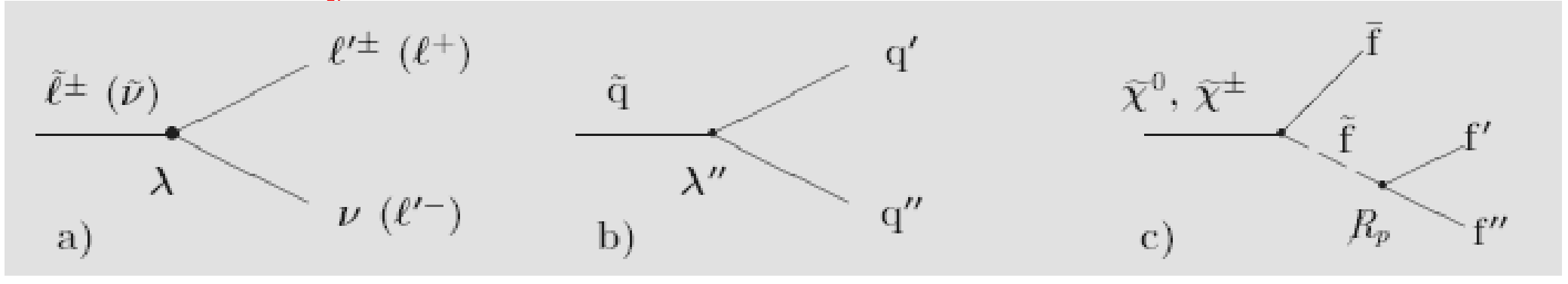

- indirect decays

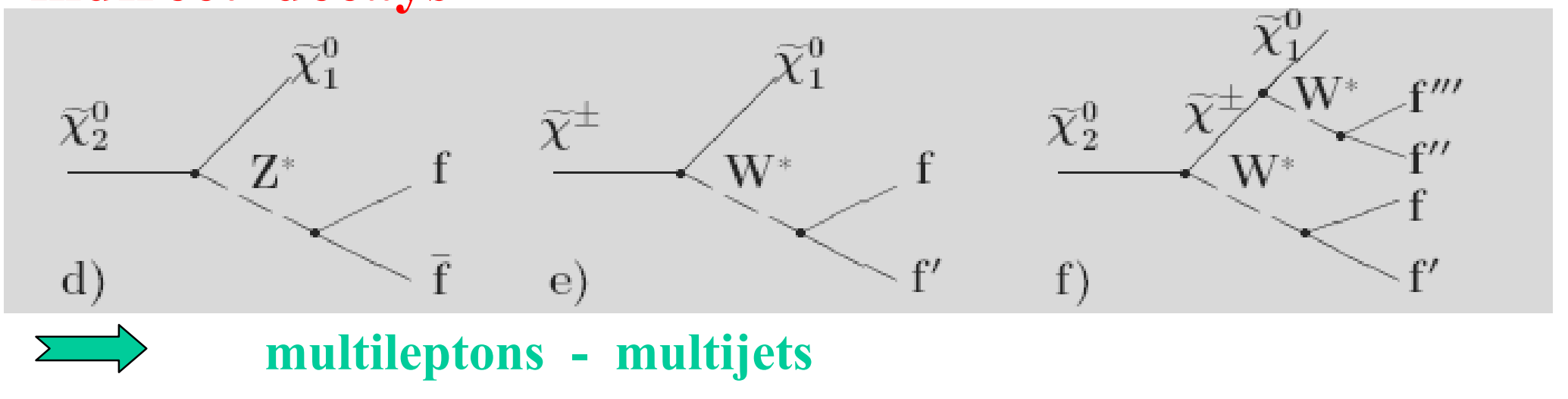


Decay Length of $\tilde{\chi}_{1}^{0}$

$L \approx \frac{1}{\lambda^{2}}\left(\frac{m_{\tilde{f}}}{100 G e V}\right)^{4}\left(\frac{1 G e V}{m_{\tilde{\chi}}}\right)^{5}$

- LEP analyses are sensitive only if the LSP has a negligible lifetime $(\mathrm{L}<1 \mathrm{~cm})$

$>m_{\widetilde{x}}>10 \mathrm{GeV}$

$\sim 10^{-5}<\lambda<10^{-2}$ up to 1

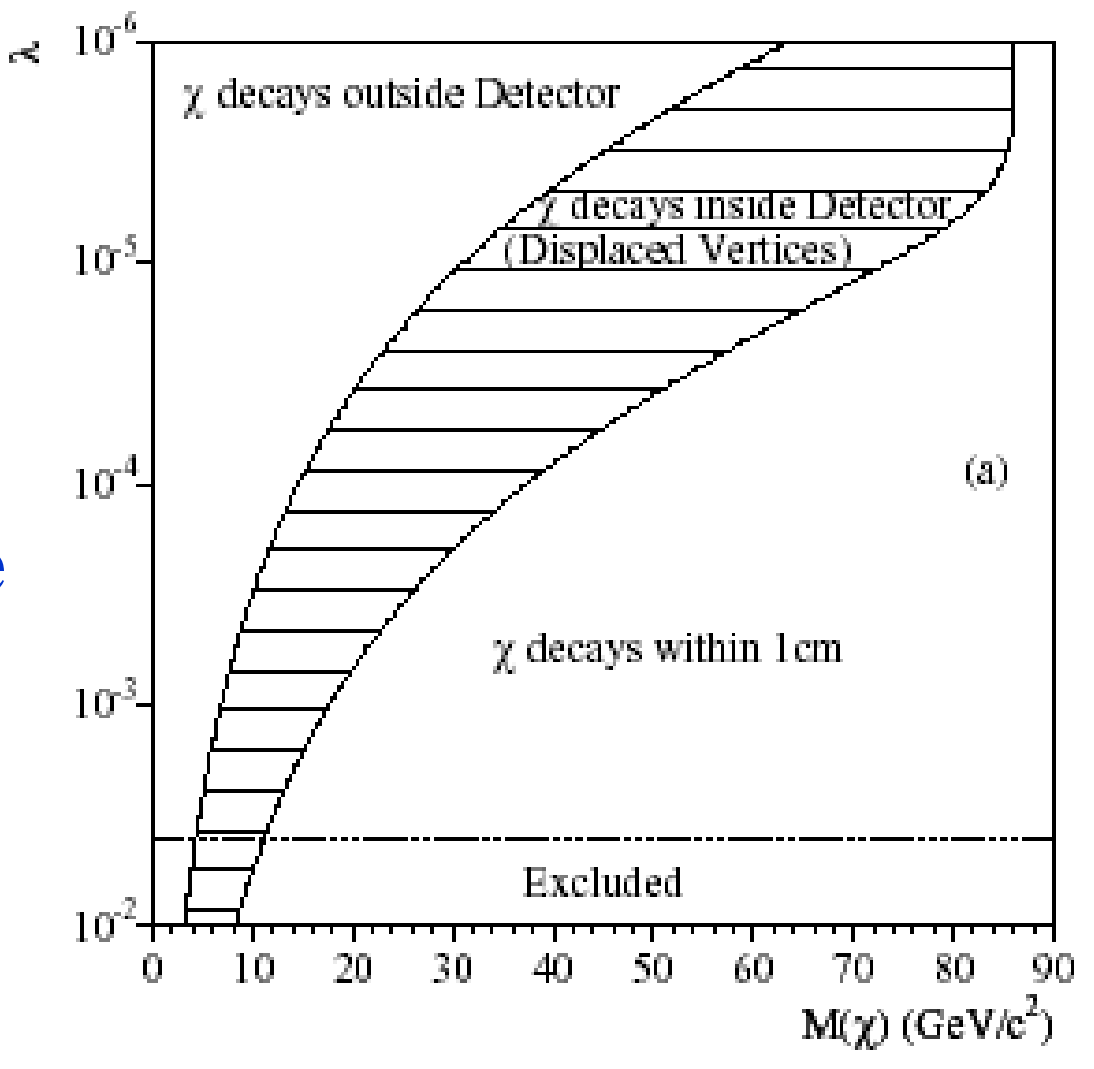

Displaced Vertices $<\lambda<$ indirect SM bounds 
$>$ Suppose one Coupling $\lambda_{\mathrm{ijk}}{ }^{\left({ }^{\prime}\right.}{ }^{\prime \prime} \neq \mathbf{0}$

$>$ Consider that many channels have to be combined

$>$ Optimize Signal selection on various topologies

--with sequential cuts : ALEPH, OPAL, L3, DELPHI (LLE $\bar{E})$

--using lepton identification and lepton isolation criteria $(\mathrm{LLE}, \mathrm{LQ} \overline{\mathrm{D}})$

-- with neural network methods : DELPHI $(\overline{\mathrm{U}} \overline{\mathrm{D}} \overline{\mathrm{D}})$

-- using jet algorithm, $b$ tagging ( $L Q \bar{D}, \bar{U} \bar{D} \bar{D})$

$>$ Calculate signal reconstruction efficiency

-- optimization on different mass combinations depending on the decays and the kinematics

$>$ If no significant deviation from the SM, set $95 \%$ CL limits on:

*cross-sections * couplings and sparticle masses * exclusion plots in the MSSM regions 
Minimal SUSY scenario :

- Topologies predicted in a Constrained MSSM =>

- gaugino mass unification ( $\left.M_{1} \approx 0.5 M_{2}\right)$ at $\mathrm{EW}$ scale

- Mass universality at GUT scale $=>\quad \mu, \tan \beta, m_{0}$

- Trilinear terms are set to $0=>A_{b, t, \tau}=0$

- Mixing angles for stop and sbottom $=>\quad \phi_{\widehat{t}}, \phi_{\widetilde{b}}$

Bounds on RpV Couplings at EW scale:

$\lambda: \sim 5.10^{-2} \quad \lambda^{\prime}: \sim 2.10^{-2}(131)$ up to $0.56(232)$

$\lambda^{\prime \prime}: \sim 0.5$ up to $\sim 1.23$ (except $\left.\lambda^{\prime \prime}{ }_{112}=\lambda^{\prime \prime}{ }_{121} \sim O\left(10^{-9}\right), \lambda^{\prime \prime}{ }_{131}=\lambda^{\prime \prime}{ }_{113}=10^{-4}\right)$

( for a sparticle mass of $100 \mathrm{GeV} / \mathrm{c}^{2}$ ) 
- LEP 2 : 5 years of data taking $\mathrm{E}_{\text {cm }}$ up to $209 \mathrm{GeV}$

$\sim 714 \mathrm{pb}^{-1}$ per experiment

\begin{tabular}{ccc} 
Year & $\mathbf{E}_{\mathrm{cm}}$ & $<\mathrm{L} / \mathrm{Exp}>$ \\
\hline $\mathbf{G e V}$ & $\mathbf{p b}^{-1}$
\end{tabular}

$1996 \quad 161-172 \sim 20$

$1997 \sim 183 \sim 55$

$1998 \sim 189 \quad \sim 170$

$1999 \quad 192-202 \sim 230$

$2000 \quad 204-209 \quad 227$

- Backgrounds :

* Four fermions $\left(Z Z W^{+} W^{-}\right)$ $f \bar{f}(\gamma)$

$\gamma \gamma$

- Signal :

generated using

SUSYGEN 2.2 (3.0 )

No evidence for a significant signal observed in all the $\mathrm{RpV}$ searches MANY LIMITS (at $95 \%$ ) are derived 


\section{Single sneutrino production}

- Resonant sneutrino $\widetilde{v}_{\mu}, \widetilde{v}_{\tau}$ production $\sigma \propto \lambda_{i j k}^{2}$ $\longrightarrow$ probes masses up to $\mathrm{E}_{\mathrm{cm}}$

\section{DELPHI}

- Single gaugino production

Analysis of final states : 3 topologies $e^{-\lambda_{1 j}} \cdot \bar{\chi}^{0} \bar{\chi}^{ \pm}$

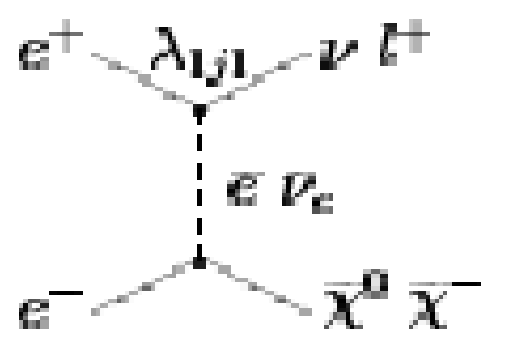

$>2$ leptons and $\mathbf{E}_{\text {miss }}$

$>4$ or 6 leptons (with or without $\mathbf{E}_{\text {miss }}$ )

$>$ leptons + jets

- Couplings $\lambda_{121}, \lambda_{131}$

limits on $\lambda$

$\rightarrow 1 .-3 \cdot 10^{-3}$
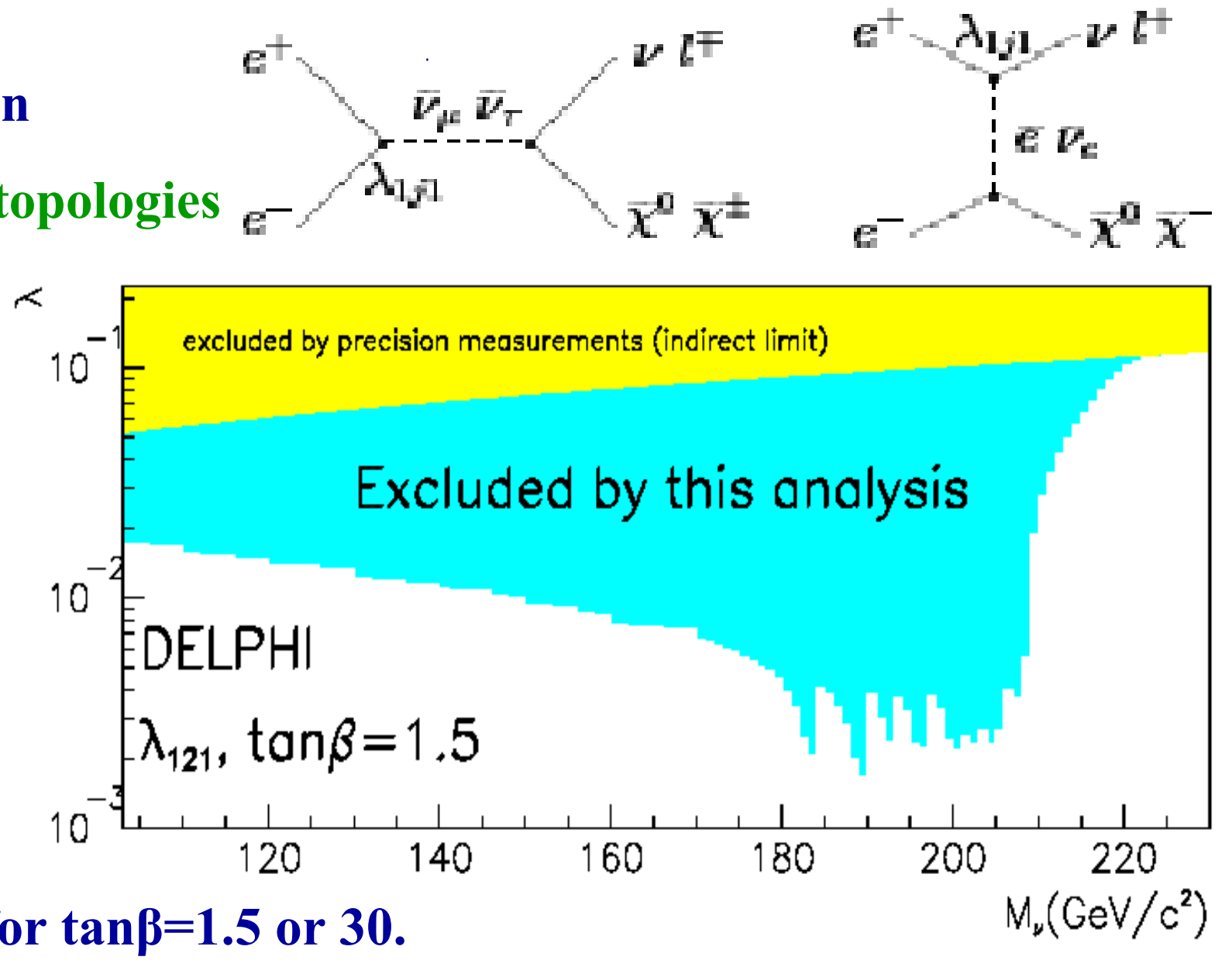


\section{Single sneutrino production}

- single sneutrino $\widetilde{v}_{e}, \widetilde{v}_{\mu}, \widetilde{v}_{\tau}$ production: $\quad e \gamma \rightarrow \widetilde{v}_{j} l_{k}$

\section{ALEPH}
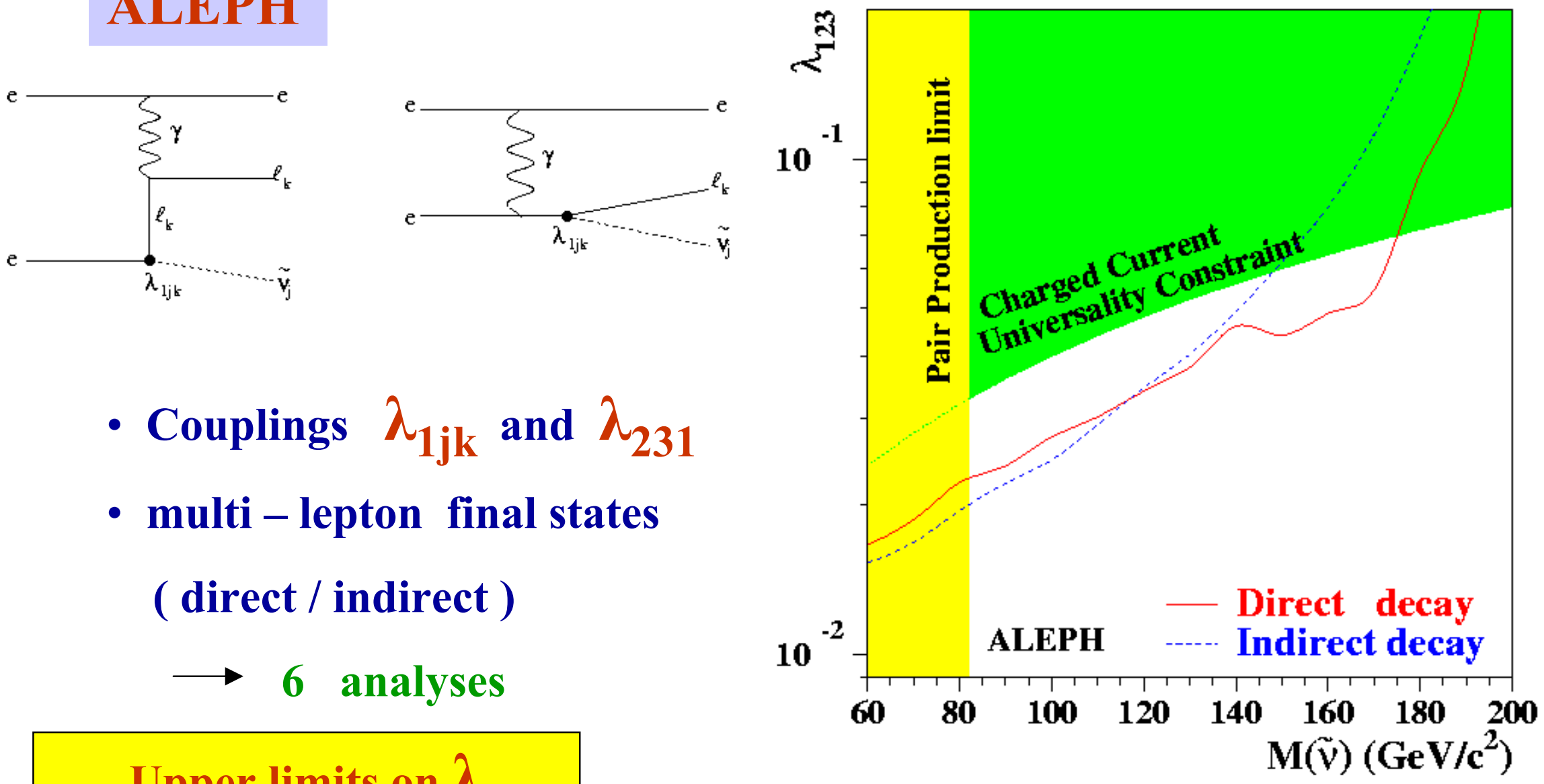

Upper limits on $\lambda$

$\sim 7 \cdot 10^{-3}-3 \cdot 10^{-2}$

( for a sneutrino mass of $100 \mathrm{GeV} / \mathrm{c}^{2}$ ) 


\section{Gaugino pair production}
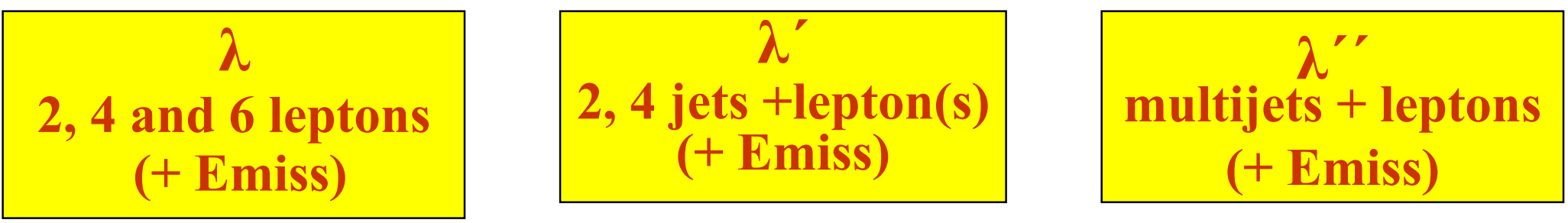

- Final states

\begin{tabular}{|c|c|c|c|}
\hline & LLE & $\mathrm{LQ} \overline{\mathrm{D}}$ & $\overline{\mathrm{U}} \overline{\mathrm{D}} \overline{\mathrm{D}}$ \\
\hline$\tilde{\chi}^{0} \tilde{\chi}^{0}$ & $4 l+\not$ & $\begin{array}{c}1,2 l+4 j+\not \\
2 l+4 j\end{array}$ & $6 j$ \\
\hline$\tilde{\chi}^{ \pm} \tilde{\chi}^{ \pm}($dir. $)$ & $2,4,6 l+\not t$ & $1,2 l+4 j+\not{H}$ & $6 j$ \\
\hline$\tilde{\chi}^{ \pm} \tilde{\chi}^{ \pm}(i n d)$. & $\begin{array}{l}\tilde{\chi}^{0} \tilde{\chi}^{0}+W W \\
\geq 4 l+n j+\not\end{array}$ & $\begin{array}{c}\tilde{\chi}^{0} \tilde{\chi}^{0}+W W \\
\geq 4 j+n l+\not\end{array}$ & $\begin{array}{c}\tilde{\chi}^{0} \tilde{\chi}^{0}+W W \\
\geq 6 j+n l\end{array}$ \\
\hline
\end{tabular}

- $\tilde{\chi}^{ \pm}$indirect decay is the dominant decay channel in almost all the MSSM parameter space 


\section{Limits from Gaugino searches}

DELPHI Scans in $\mu, M_{2}$
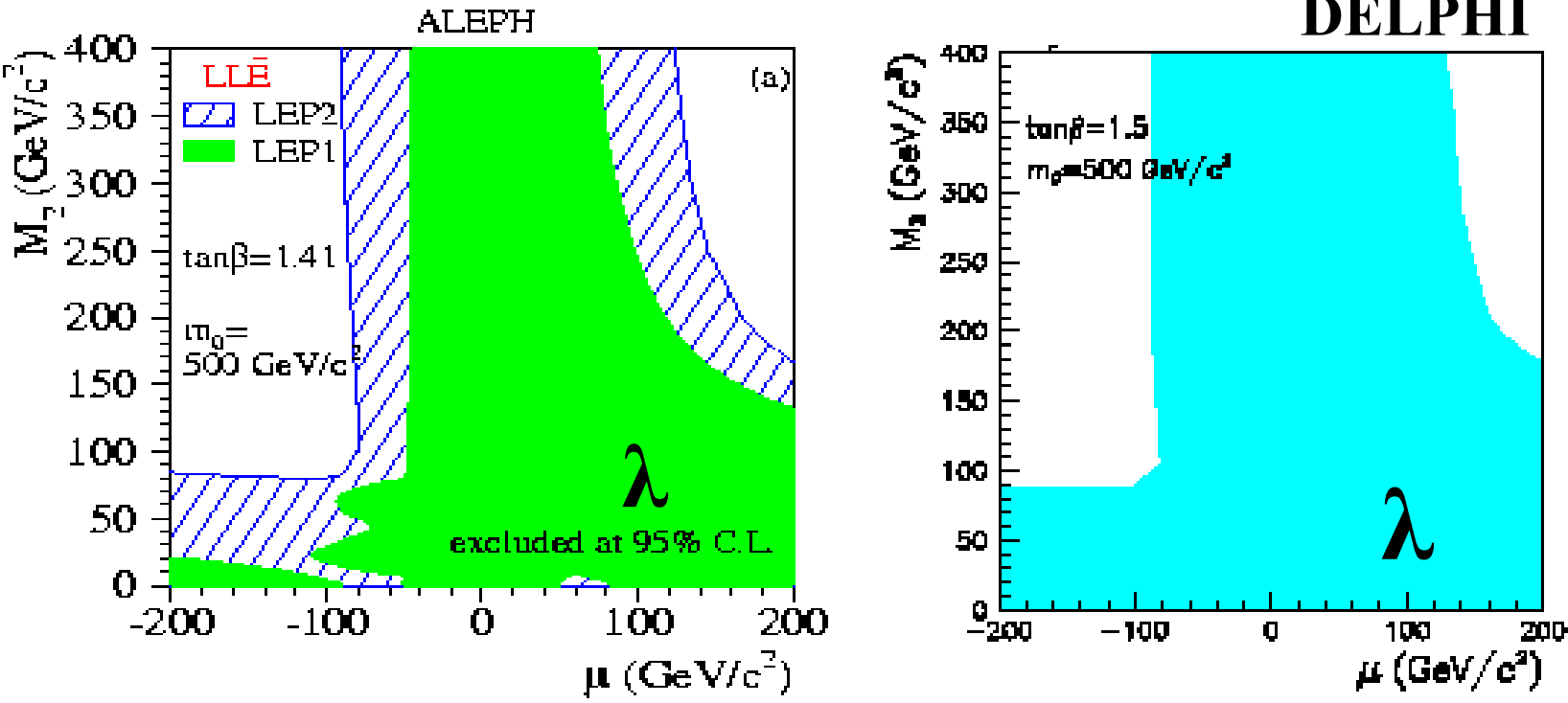
for different values of $\mathbf{m}_{0}$ and $\tan \beta$



\section{DELPHI}

\section{NEW}

- Mass limits at $95 \% \mathrm{CL}$ in $\mathrm{GeV} / \mathrm{c}^{2}$

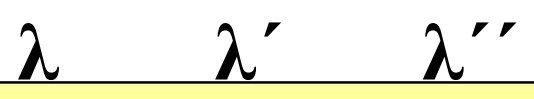

\begin{tabular}{|c|c|c|c|}
\hline & 40.2 & - & 39.9 \\
\hline D & 39.5 & - & 38.0 \\
\hline & 84.0 & - & 80.0 \\
\hline & 107.2 & - & 107.2 \\
\hline & 103 & 103 & 103 \\
\hline U & 103 & & 102.5 \\
\hline L & 103.0 & - & 102.7 \\
\hline
\end{tabular}
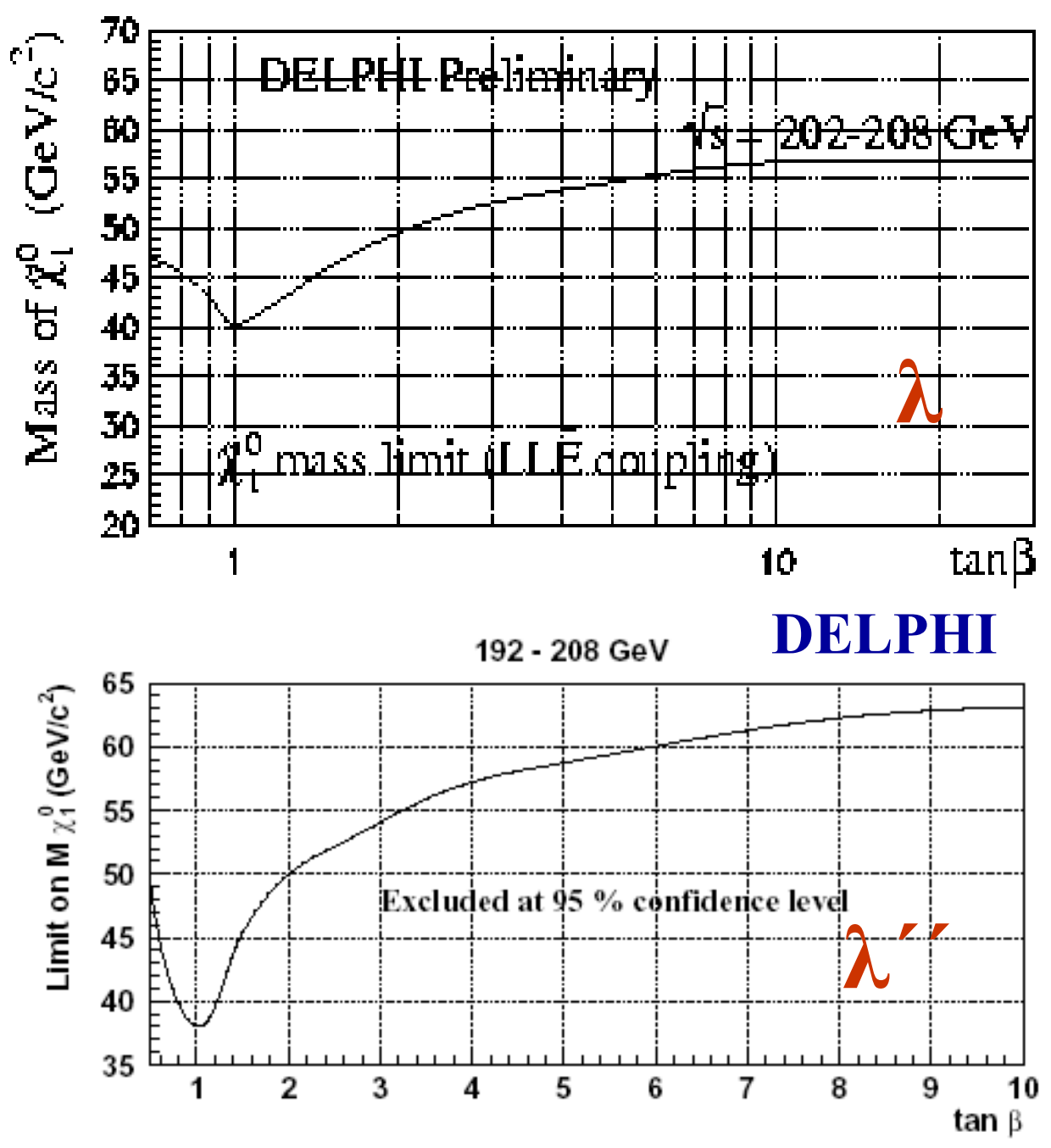


\section{slepton pair production}

\section{$\widetilde{e}, \widetilde{\mu}, \widetilde{\tau}$}

- Direct decay : $\quad e^{+} e^{-} \rightarrow \tilde{l}^{+} \tilde{l}^{-} \rightarrow 4$ fermions

- Indirect decay : $e^{+} e^{-} \rightarrow l^{+} l^{-} \tilde{\chi}_{1}^{0} \tilde{\chi}_{1}^{0} \rightarrow 8$ fermions
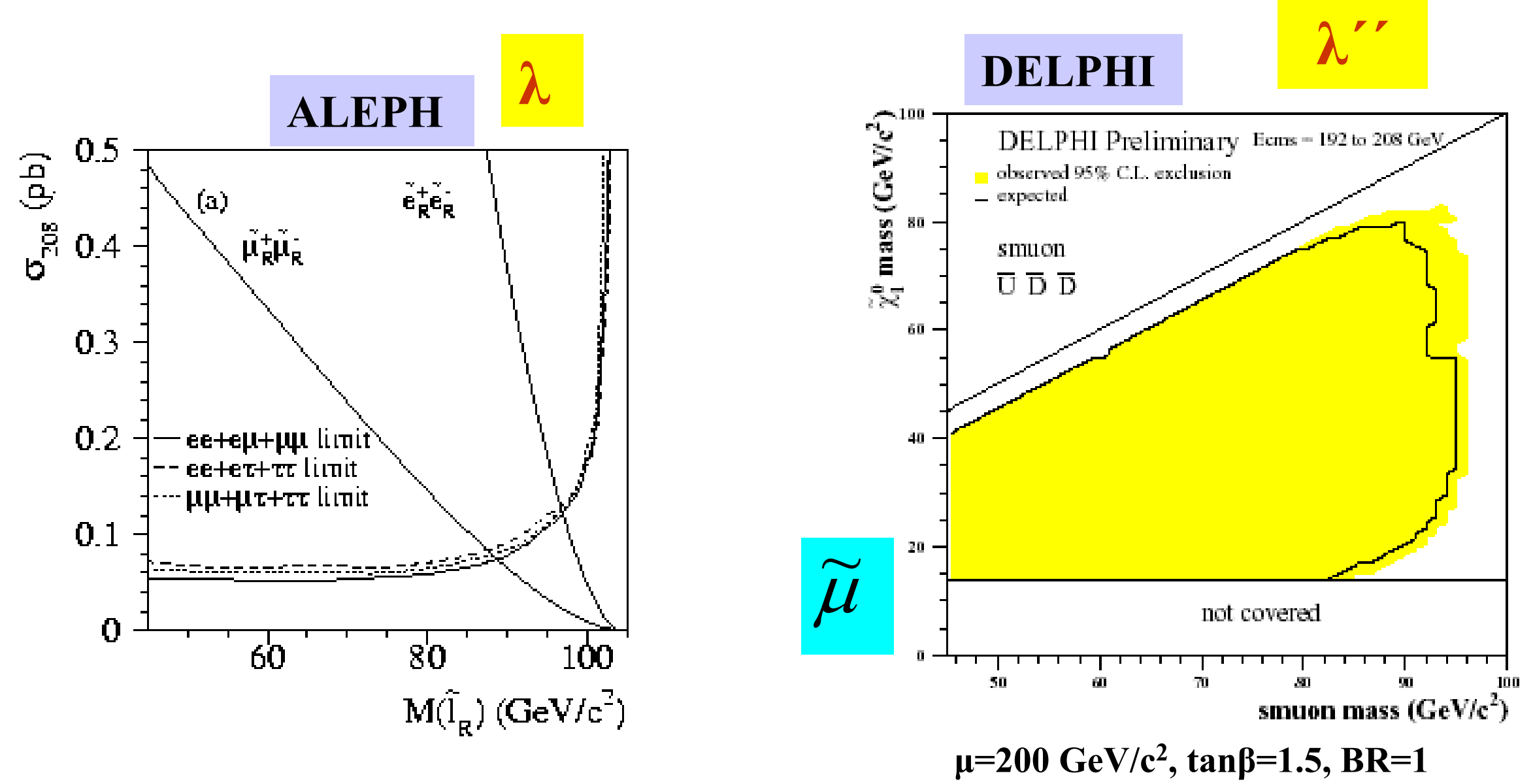


\section{slepton pair production}
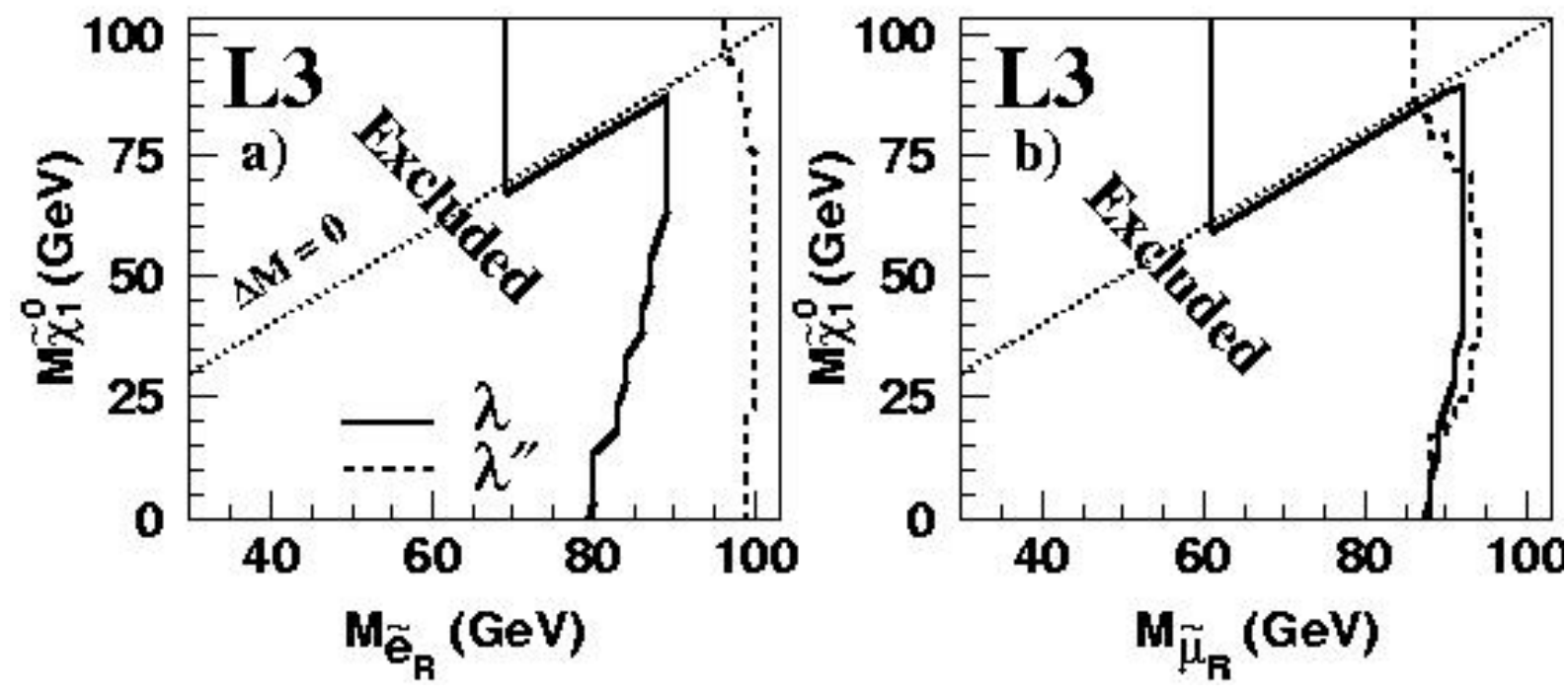

- MSSM exclusion contours, at $95 \%$ C.L.
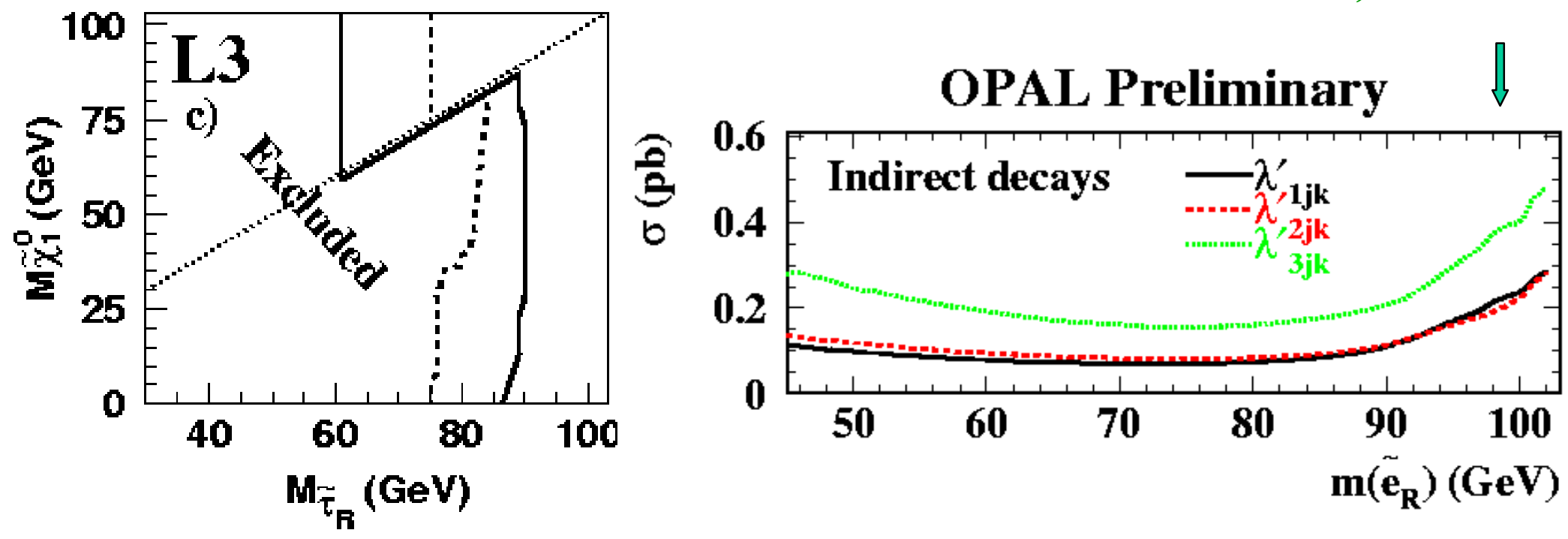


\section{Sneutrino pair production}

$$
\begin{aligned}
& \widetilde{v}_{e}, \widetilde{v}_{\mu}, \widetilde{v}_{\tau} \cdot \text { Direct decay : } \quad e^{+} e^{-} \rightarrow \widetilde{v} \widetilde{\bar{v}} \rightarrow 4 \text { fermions } \\
& \text { - Indirect decay : } e^{+} e^{-} \rightarrow v \bar{v} \widetilde{\chi}_{1}^{0} \tilde{\chi}_{1}^{0} \rightarrow 8 \text { fermions }(2 v)
\end{aligned}
$$

\section{ALEPH}

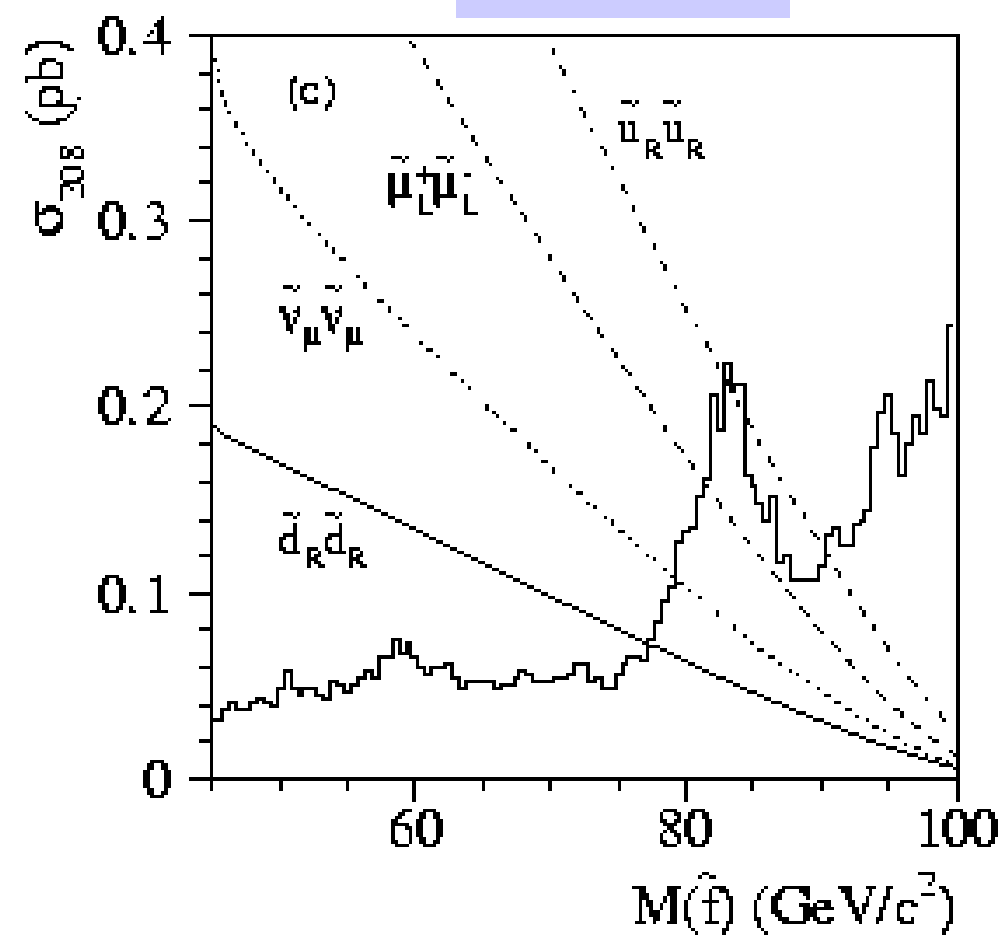

Excluded $\widetilde{v} \widetilde{v}$ cross-section ( 4 jets )

\section{DELPHI}

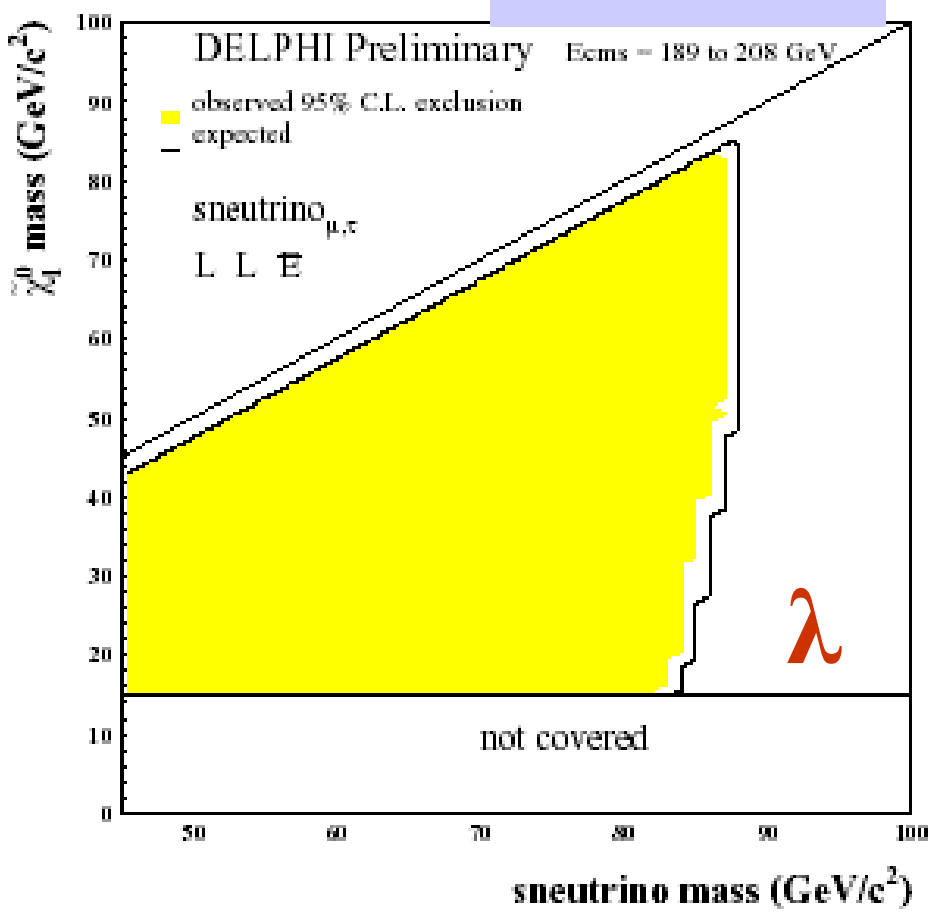

Excluded contour , at 95\% CL for $\widetilde{v}_{\mu}, \widetilde{v}_{\tau}$ for indirect decays 
OPAL Preliminary

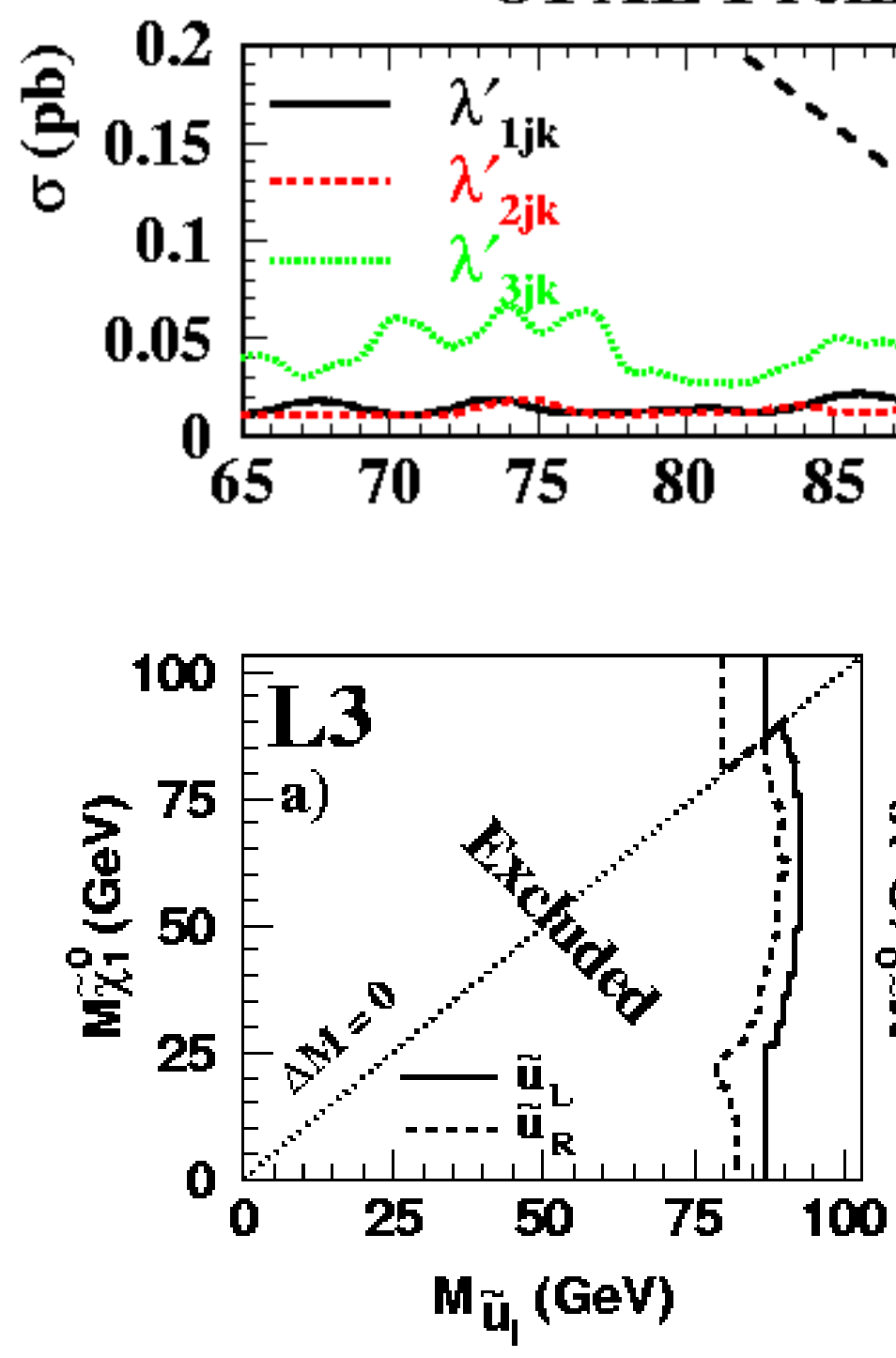

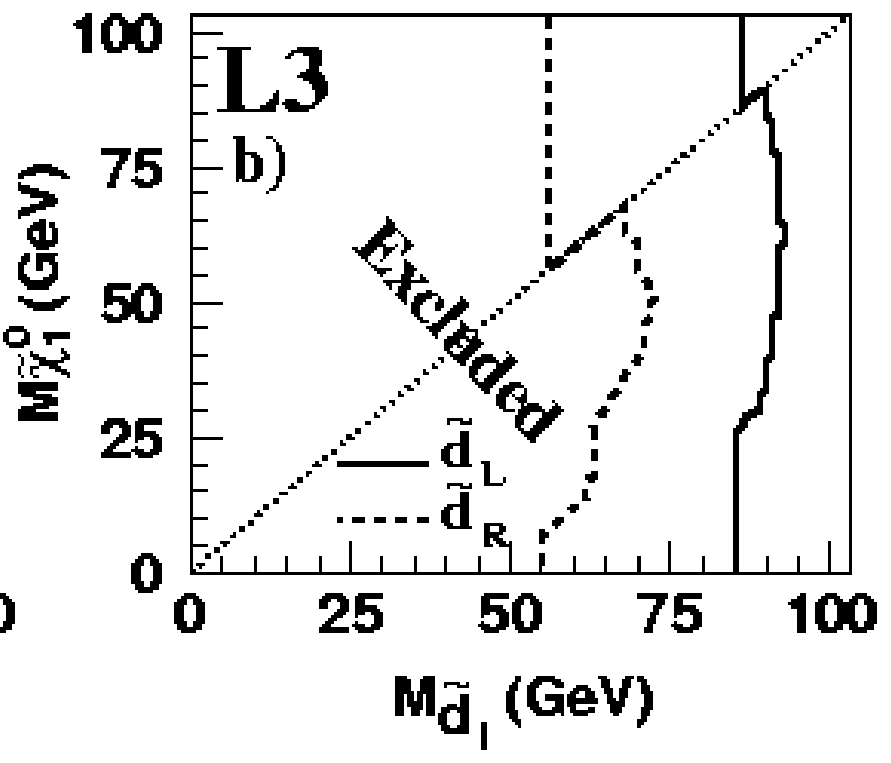

$\lambda^{\prime \prime}$

\section{MSSM}

Exclusion contours, at $95 \% \mathrm{CL}$
- Direct decays :

$=>\widetilde{q} \underset{q}{q}=>4$ fermions

- Indirect decays :

$=>q \bar{q} \widetilde{\chi}_{1}^{0} \widetilde{\chi}_{1}^{0}=>8$ fermions 
$\widetilde{t}, \widetilde{b}$

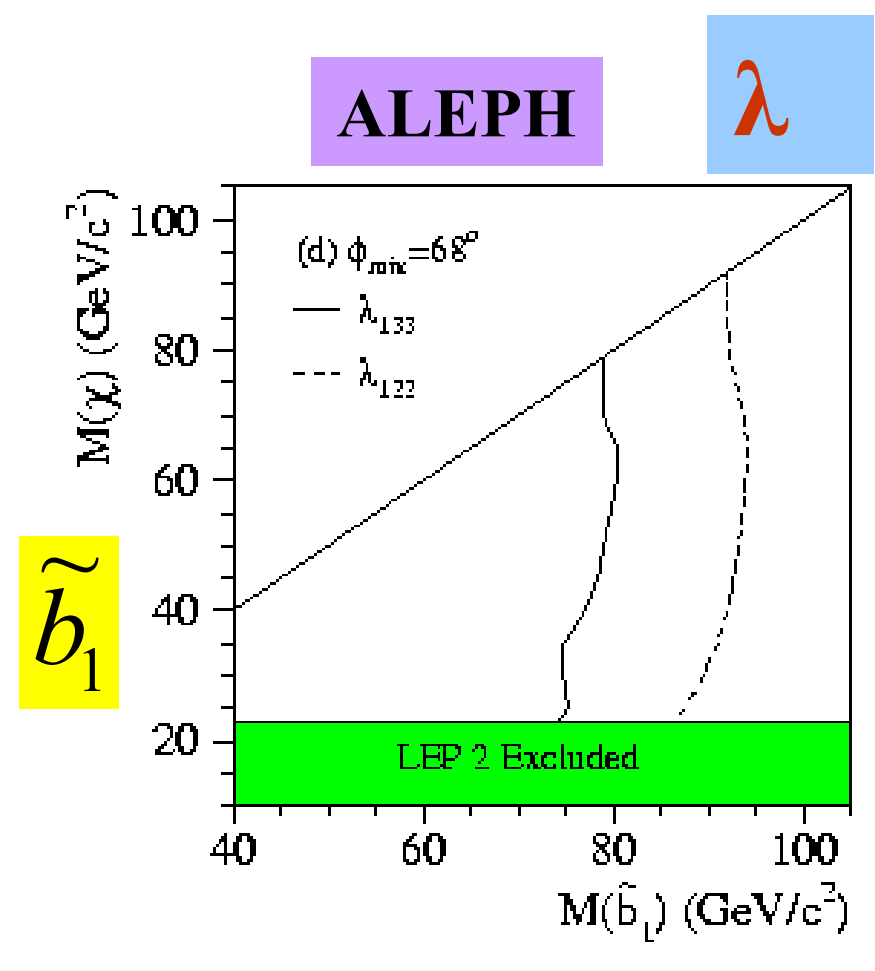

- Mixing : $\widetilde{t}_{1}=\widetilde{t}_{L} \cos \phi_{\widetilde{q}}+\widetilde{t}_{R} \sin \phi_{\widetilde{q}}$

\section{DELPHI}

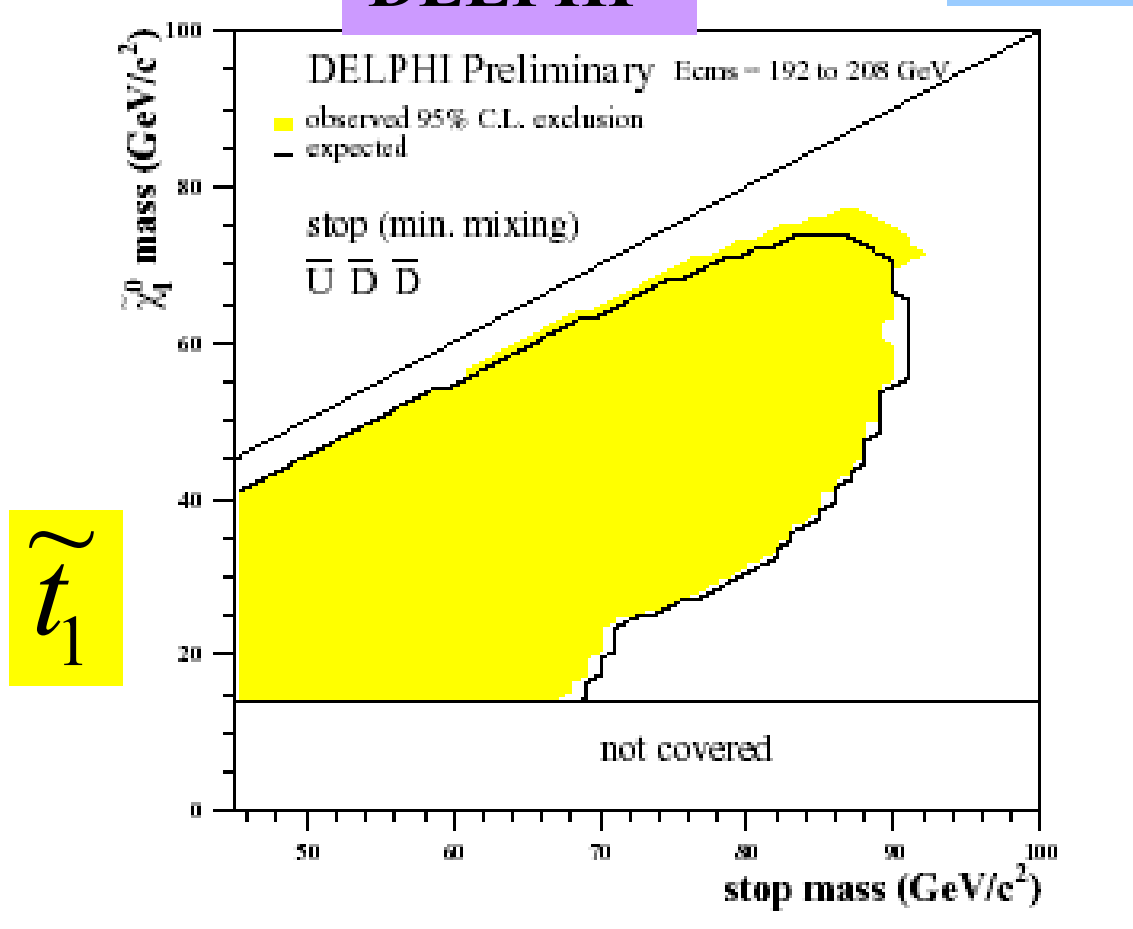

$\phi_{\widetilde{b}}=68^{0} \quad$ Exclusion contours at 95\% CL $\quad \phi_{\widehat{t}}=56^{0}$ Mixing angle for vanishing coupling to $\mathrm{Z}$ for $\tilde{t}, \tilde{b}=\boldsymbol{\sigma}$ min 


\section{Single top production}

$e^{+} e^{-} \rightarrow t \bar{c}(\bar{u}) \rightarrow q q^{\prime} b \bar{c}(\bar{u})$

$$
e^{+} e^{-} \rightarrow t \bar{c}(\bar{u}) \rightarrow l v b \bar{c}(\bar{u})
$$

\section{DELPHI Preliminary}

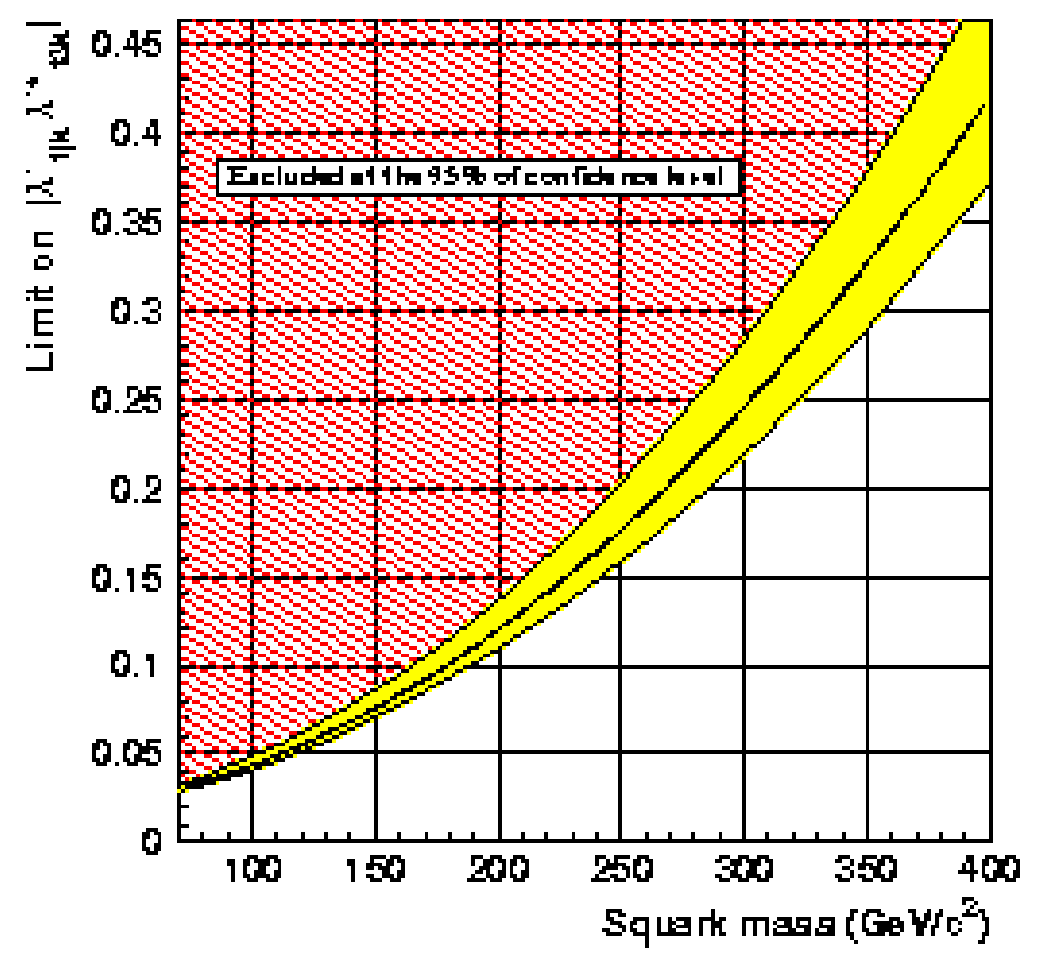

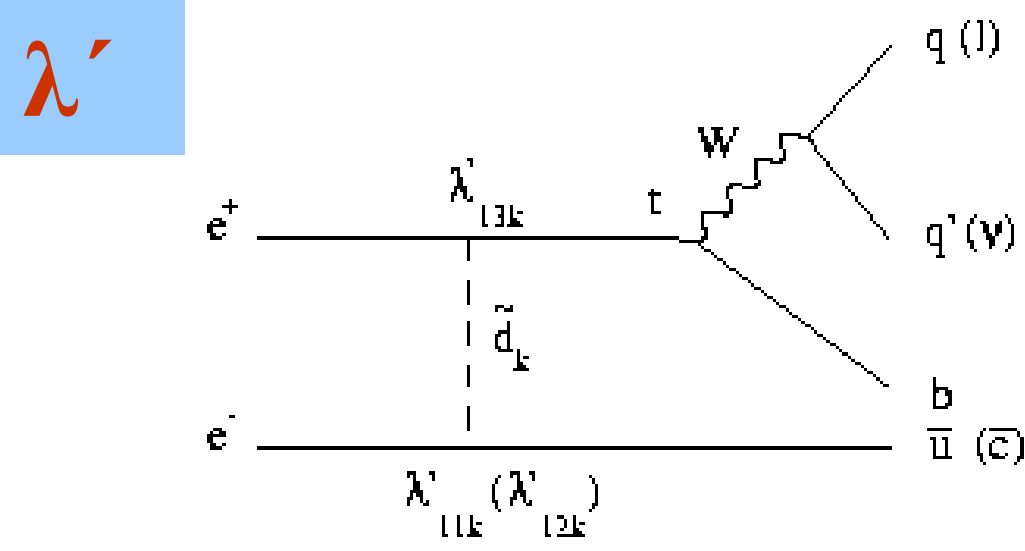

- Analysis with neural network method

- b tagging used

$\sigma_{\text {upper limit }}=0.11 \mathrm{pb}$

( $\sigma$ upper limit at $95 \%$ CL ) 


\section{LSP $\tilde{\chi}_{1}^{0}$ Mass limits}

- Limits are set at $95 \%$ CL via a dominant Coupling LLE $\bar{E} \quad L Q \bar{D} \quad \bar{U} \bar{D} \bar{D}$ $\lambda_{133}$ or $\lambda_{311}^{\prime}$ or $\lambda^{\prime \prime}{ }_{223}$ $60.2 \quad 44.2 \quad 42.2 \quad\left(\mathrm{GeV} / \mathrm{c}^{2}\right)$

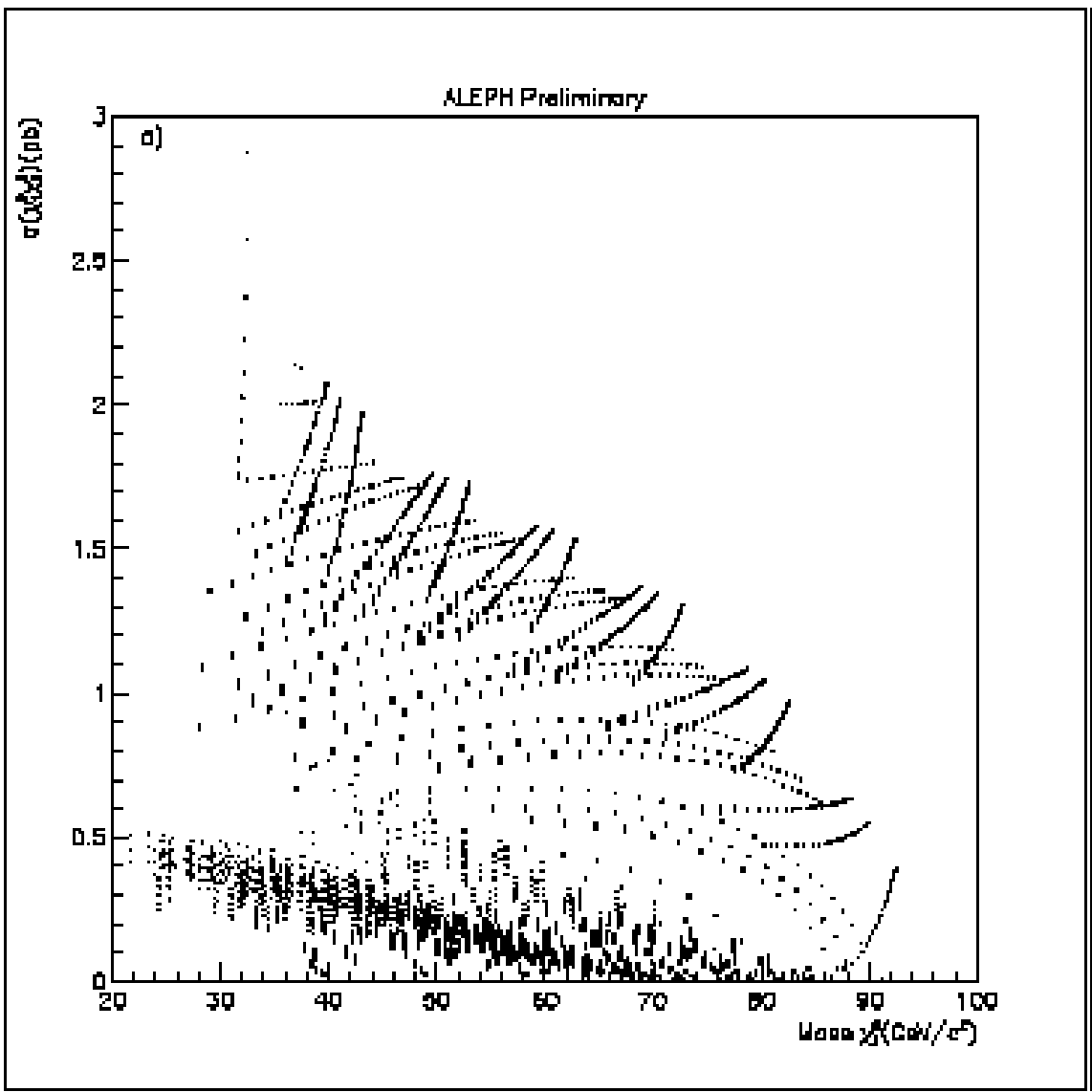

- production cross-section of $\quad \tilde{\chi}_{1}^{0}$ ALEPH PRELIMINARY vrs mass in all $\mu$, all $M_{2} \tan \beta(2-4)$, $\mathrm{m}_{0}(200-500) \mathrm{GeV} / \mathrm{c}^{2}$ 
New lower mass Limits $\left(\mathrm{GeV} / \mathrm{c}^{2}\right)$

ADLO

\begin{tabular}{|c|c|c|c|c|c|c|c|c|c|c|c|c|}
\hline sfermion & \multicolumn{3}{|c|}{$\lambda($ dd) } & \multirow{2}{*}{$\frac{\lambda^{\prime} \text { (dd) }}{(89)}$} & \multirow{2}{*}{$\frac{\lambda^{\prime \prime}(\text { dd) }}{96}$} & \multicolumn{3}{|c|}{$\lambda$ (id) } & \multicolumn{2}{|c|}{$\lambda^{\prime}$ (id) } & \multicolumn{2}{|c|}{$\lambda^{\prime \prime}$ (id) } \\
\hline$\widetilde{e}_{R}\left(\widetilde{e}_{L}\right)$ & 96 & 69 & (89) & & & 9675 & $\begin{array}{l}9 \\
99\end{array}$ & 95 & 939 & 92 & $\begin{array}{ll}94 & 96 \\
\end{array}$ & 92 \\
\hline$\tilde{\mu}_{R}\left(\widetilde{\mu}_{L}\right)$ & 87 & 61 & (74) & (81)(77) & 86 & \begin{tabular}{l|l}
96 & 8 \\
\end{tabular} & 3792 & 90 & $\begin{array}{ll}90 & 8 \\
\end{array}$ & 87 & \begin{tabular}{l|l|}
85 & 86 \\
\end{tabular} & 87 \\
\hline$\tilde{\tau}_{R},\left(\tilde{\tau}_{L}\right)$ & 87 & 61 & (74) & (76) & 75 & 958 & 690 & 90 & $76-$ & - & - 75 & - \\
\hline$\widetilde{v}_{e}$ & 100 & 95 & 90 & 90 & 99 & 9895 & 995 & 98 & \begin{tabular}{l|l}
91 & 8
\end{tabular} & 88 & \begin{tabular}{l|l}
88 & 99
\end{tabular} & - \\
\hline$\widetilde{v}_{\mu}$ & 90 & 65 & 76 & \begin{tabular}{l|l}
79 & 75
\end{tabular} & 70 & \begin{tabular}{l|l}
89 & 78
\end{tabular} & 881 & 85 & $78-$ & - & \begin{tabular}{l|l}
65 & 70
\end{tabular} & - \\
\hline$\tilde{v}_{\tau}$ & - & 65 & 76 & 75 & 70 & 8978 & 881 & 85 & 78 & & \begin{tabular}{l|l|}
65 & 70 \\
\end{tabular} & - \\
\hline$\tilde{t}\left(\tilde{t}_{L}\right)$ & - & - & - & (97) & - & (91) & ( & (92) & (85) & & (71.5) & (87) \\
\hline$\tilde{b}\left(\tilde{b}_{L}\right)$ & - & - & - & - & - & (90) & & & (80) & & (71.5) & (78) \\
\hline$\tilde{u}_{R}\left(\tilde{u}_{L}\right)$ & - & - & - & - & $\begin{array}{r}(82.5) \\
80(87) \\
\end{array}$ & & - & & - & & $79(87)$ & \\
\hline$\widetilde{d}_{R}\left(\tilde{d}_{L}\right)$ & - & - & - & - & $\begin{array}{r}(77) \\
56(86)\end{array}$ & & - & & - & & $55(86)$ & \\
\hline
\end{tabular}


- RpV has inspired new interesting scenarios of SUSY searches

- RpC and RpV are two complementary ways of SUSY searches

- Searches for SUSY with RpV performed by all LEP collaborations ( ADLO) in ...many channels ....

- No evidence for SUSY with RpV so far at LEP

- Limits on SUSY particles and RpV Couplings are set at 95\% CL

- Limits from RpV searches are comparable with the RpC ones! 
..... Many papers and ... many searches on SUSY !

..... but there is NO evidence up to now

$>$ SUSY with RpV

predicts very clear signatures especially for couplings

with $\quad \Delta L \neq 0 \quad(L L \bar{E}, L Q \bar{D})$

all hopes are shifted

towards future colliders ! 